\title{
RESEARCH OF VIBROACOUSTIC INTERFERENCE OCCURRING ON UAV
}

\author{
S. Grzywiński *, S. Żygadło **
}

\begin{abstract}
The article presents the results of research on vibroacoustic disturbances registered on UAV (Unmanned Aerial Vehicle) occurring in real conditions of shock wave measurement by an acoustic locator mounted on the aircraft wing. The attention was focused on determining the parameters of disturbances affecting the process of detecting and determining the coordinates of artillery projectiles flying at supersonic speeds. The main emphasis was placed on characterizing, investigating and analyzing disturbances in the form of acoustic noise and vibration of the UAV structure.
\end{abstract}

Keywords: acoustic location of projectiles, vibroacoustic disturbances, mechanical vibrations, noise

\section{Introduction}

One of the possible solutions that significantly increase the operational capabilities of the locator acoustic system designed to detect and determine the coordinates of artillery projectiles is its implementation on unmanned aerial vehicle - UAV. Nevertheless, the acoustic locator of artillery projectiles installed on the UAV wing is exposed to a number of phenomena that can significantly reduce the effectiveness of projectile detection for the assumed detection range. Due to the band of recorded signals and the structure of the sensors used, special attention should be paid to vibroacoustic disturbances. In the following case these are mainly noise and vibrations introduced by the UAV as well as the acoustic background prevailing during actual measurement conditions.

\section{Characteristics and research of vibroacoustic disturbances}

Analyzing the interference emitted by the UAV, one can distinguish many elements and systems of its mechanical construction which are sources of the occurring disturbances. Vibroacoustic disturbances can have a dual generation mechanism: mechanical and aerodynamic. In both cases, the resonant frequencies of the mechanical-acoustic part of the UAV structure are an important parameter, since any mechanical vibrations of the aircraft construction and the generated noise will transfer to the acoustic sensor membranes, worsening the measurement conditions through reducing the signal to noise ratio. The basic source of mechanical disturbances is the $n$-periodic, quasi-stationary noise and engine vibrations that can be divided into:

- aerodynamic noise in the suction and exhaust ducts, created during periodic opening and closing of the free gas outlet into the atmosphere,

- the gasodynamic process in the cylinders that is the reason for their vibrations, as well as the infiltrating noise,

- mechanical forces inducing vibrations caused by the reciprocating movement of the pistons in the engine crankshaft.

Stanisław Grzywiński, PhD.: Faculty of Mechatronics \& Aerospace, Military University of Technology, Urbanowicza 2, 00-908 Warsaw, PL, stanislaw.grzywinski@wat.edu.pl

** Stanisław Żygadło, PhD.: Faculty of Mechatronics \& Aerospace, Military University of Technology, Urbanowicza 2, 00-908 Warsaw, PL, stanislaw.grzywinski@wat.edu.pl 
All combustion piston engines are characterized by pulsation of torque depending on the number of cylinders, while unbalanced inertia forces depend on the system implementation of cylinders. Piston engines are the largest concentrated mass, and their unbalanced forces are transferred to the UAV structure resulting in complex, multimodal mechanical vibrations. The noise amplitude spectrum contains characteristic frequency components and their harmonics related to the number of engine revolutions $n$ and the number of cylinder $s$. The formula describing the frequency components of the disturbance spectrum can be written as follows:

$$
f_{i}=i \cdot t_{\mathrm{s}} \cdot \frac{n_{o} \cdot s}{60}
$$

where:

$f_{\mathrm{i}} \quad$ - ith number of harmonic amplitude spectrum, $\mathrm{i}=1,2,3, \ldots$

$n_{\mathrm{o}} \quad$ - number of engine revolutions;

$s \quad$ - number of cylinders;

$t_{\mathrm{s}} \quad$ - a constant describing the type of engine.

The mechanism of generating aerodynamic noise is connected with the motion of the medium, which causes vibroacoustic disturbances, mainly concerning the following phenomena:

- $l \cdot n$ - periodic, stationary noise from the propeller blades ( $l$ - number of propeller blades);

- $s \cdot n$ - periodic vibrations of the structure;

- fluctuation of air streams.

The number of blades has a significant influence on the noise level from the propeller blades and vibrations generated on the rotor and transferred to the UAV structure. Vibrations on the rotor arise as a result of variable aerodynamic forces acting on the rotor, flow of rotor blades through non-uniform air flow and interference of vortex traces coming off the blades. In both cases (noise and vibrations of the structure), the interference signal can be treated as a broadband spectrum consisting of a series of rotating harmonics $f_{\mathrm{oi}}$ and harmonics from propeller blades $f_{\mathrm{li}}$ described by relations (2) and (3). The rotational frequencies and their harmonics are the result of the aerodynamic (structural or operational) imbalance of the rotor (e.g. differences in the length or spacing of the blades). Harmonics from propeller blades are the result of the portion flow of the air stream.

$$
\begin{gathered}
f_{\mathrm{oi}}=\frac{i \cdot n_{o}}{60} \\
f_{\mathrm{li}}=\frac{i \cdot l \cdot n_{o}}{60}
\end{gathered}
$$

where:

$$
\begin{aligned}
& \mathrm{i}=1,2,3, \ldots . \\
& l \quad \text { - number of propeller blades. }
\end{aligned}
$$

The main component of acoustic background noise prevailing during real measurement conditions is the variable within wide limits of disturbances caused by gusts of wind. From an acoustic point of view, the phenomena associated with wind gusts are difficult to describe due to the complicated combination of laminar and turbulent air flow. The estimated data, on the basis of which it can be assumed that for wind speed of $2 \mathrm{~m} / \mathrm{s}$ the maximum sound intensity can be about $80 \mathrm{~dB}$ for $50 \mathrm{~Hz}$, while for $5 \mathrm{~m} / \mathrm{s}$ we record $10 \mathrm{~dB}$ increase in the sound level, can be found in the literature (Panofsky, 1984). It should be noted that the frequency band of the noise moves towards higher frequencies as the wind speed increases. It can be assumed that the spectrum ranges from $20 \mathrm{~Hz}$ to $150 \mathrm{~Hz}$. In order to determine the resultant power of disturbance signals, noise and vibrations introduced by the UAV as well as the acoustic background should be taken into account. Hence, assuming the additivity of disturbance signals, marking as $L_{I n}$ the intensity level of the nth sound source, can be written a formula describing the resultant sound intensity.

$$
L_{\text {It }}=10 \log \left(\sum_{I=1}^{n} 10^{0.1 \cdot L_{I n}}\right)
$$

Analytical determination of the power of individual components of the amplitude spectrum of vibroacoustic disturbances is a very complex task which depends on many factors, including material and construction of UAV and acoustic locator. The approximation of the disturbance signal parameters 
distribution using the formulas (1) $\div$ (4) in the problems of the analysis of their impact on the effectiveness of the projectile detection by the acoustic locator is insufficient. Verification of the impact of disturbances requires complex and extensive experimental tests in which actual disturbance signals will be recorded. Selected for UAV research is characterized by the following parameters:

- engine speed $n_{o}=6000$ - $6500 \mathrm{rpm}$;

- biplane propeller (number of propeller blades) $l=2$;

- engine power $8.5 \mathrm{~kW}$;

- cruising speed approx. $140-160 \mathrm{~km} / \mathrm{h}$.

During the tests a series of measurements was carried out, which allowed to determine the values of vibration parameters and acoustic emission at the location of the potential attachment of the locator's sensors. In research, attention was paid to the noise and vibrations emitted by the UAV and to the background of the acoustic environment, i.e.:

- $n$-periodic, stationary motor noise;

- $2 n$-periodic, stationary noise from the propeller blades ( $l$ - number of propeller blades);

- complex, multi-modal mechanical vibrations of the structure;

- fluctuations of air streams and gusts of wind.

In order to record measurement data allowing for time-frequency analysis of interference signals, a remote signal recorder was designed (Rodzik, 2014, Grzywiński, 2016, 2018). It has been configured to record signals from two 1/4" wide-band condenser microphones, type 4135, and a B \& K type 4524B accelerometer. The sensors are placed on the tip of the wing. During the tests, UAV took off from the airport and made a flight on the planned route. Based on the preliminary analysis of recorded signals, 4 characteristic time intervals were identified in which different disturbance conditions occurred. In the first pre-start interval, the level of background noise was recorded without the engine running (1). The second one is the signal of a running engine (2), where the plane was on the runway. The next stage was taxiing on the runway, during which three quick changes of the engine throttle position were made (3). The last one was a flight of 100 to 600 meters (4). First of all, the recorded vibroacoustic disturbances occurring under the conditions of the running engine were analyzed (stage 2). A significant increase in the signal energy in relation to the background noise recorded on the airport strip with the engine turned off was observed. Fig. 1 presents signals of engine noise and mechanical vibrations of structures, which were recorded using the microphone 1 and the accelerometer.

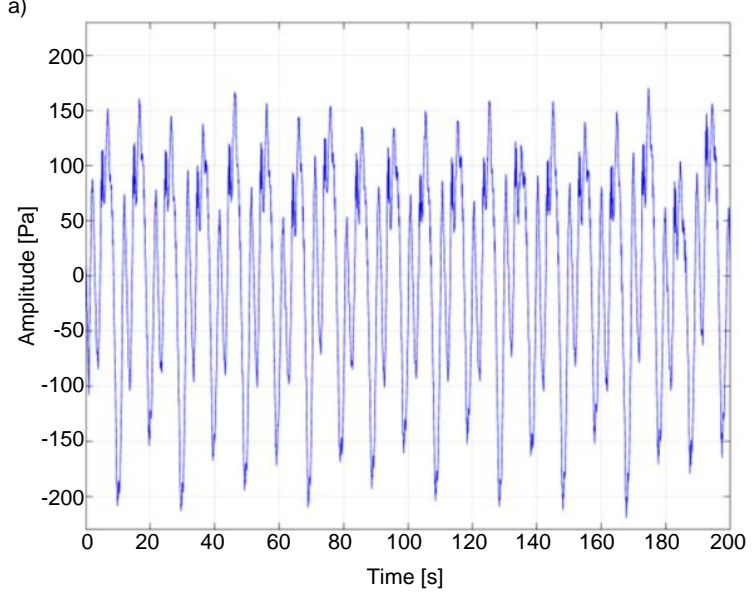

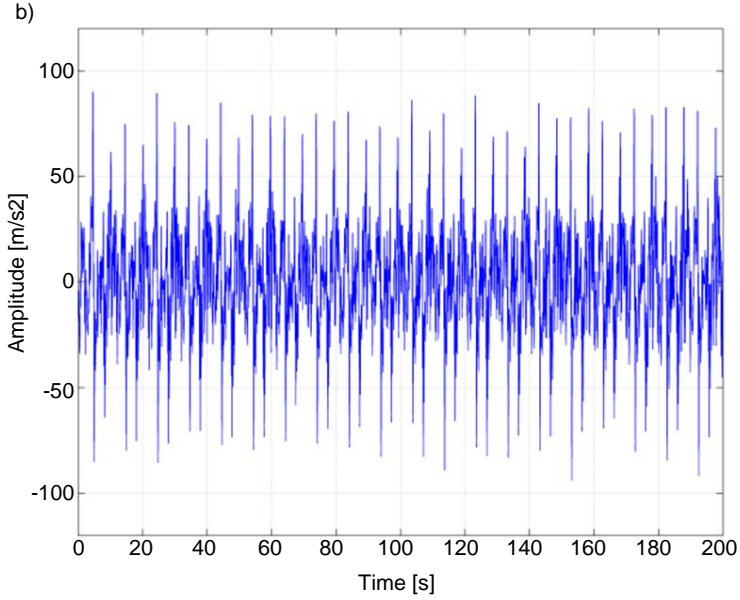

Fig. 1. Recorded noise - a) microphone 1, b) accelerometer

Time analysis of two signals has proved that both signals are correlated with the period resulting from the basic frequency of stationary engine vibrations. In addition, the recorded signal from the microphone is an asymmetrical signal, which can be caused by the additivity of individual components of the spectrum of disturbances of various phases. Fig. 2 shows the FFT spectra of recorded vibroacoustic signals. Figure 2 (a) shows the spectral components of the $n$-period engine noise and $2 n$-period noise from the propeller blades. The frequencies of the basic components of the amplitude spectrum are $100 \mathrm{~Hz}$ and $200 \mathrm{~Hz}$, which according to the formulas (2) and (3) corresponds to $6000 \mathrm{rpm}$ of the engine. This value confirms the position of the throttle set during testing, giving the average speed of rotation maintained during the 
UAV flight. On the basis of the amplitude-frequency analysis of the accelerometer signal Fig. 2 (b), the frequency of the fundamental component of the mechanical vibration spectrum of the structure was obtained, which is theoretical for the two-stroke engine described by the formula (1). Further analysis confirms the occurrence of signal harmonics at frequencies $n f_{1}$, where $\mathrm{n}=2,3,4, \ldots$. In the case of the basic spectral component for the microphone 1, the signal energy level was approx. $130 \mathrm{~dB}$, while for 3.7 $\mathrm{kHz}$ approx. $15 \mathrm{~dB}$ less. It should be noted that the level of amplitudes of the spectral components of the signal contained in the $3.5 \div 4.5 \mathrm{kHz}$ band reaches values in the order of $115 \mathrm{~dB}$. Amplitude values of the components of the motor vibration spectrum are irregular, which results from the different attenuation of individual components by the UAV structure.

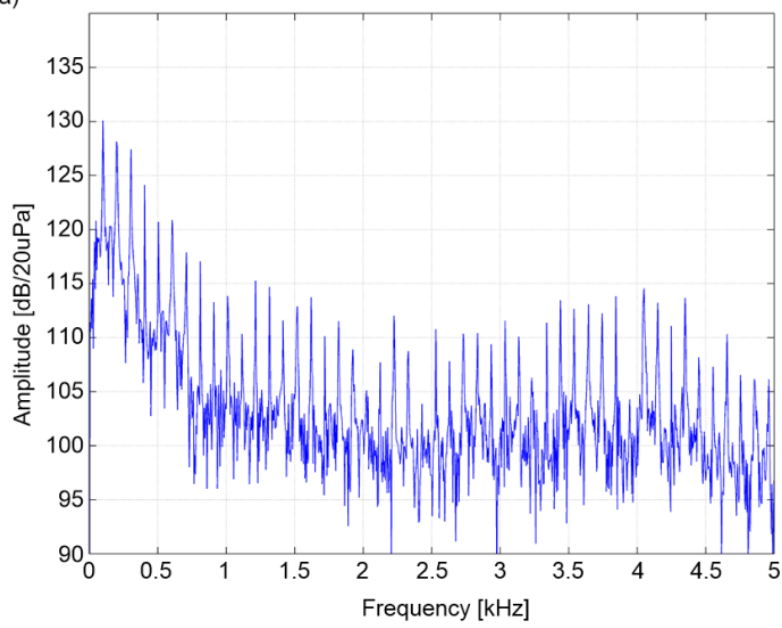

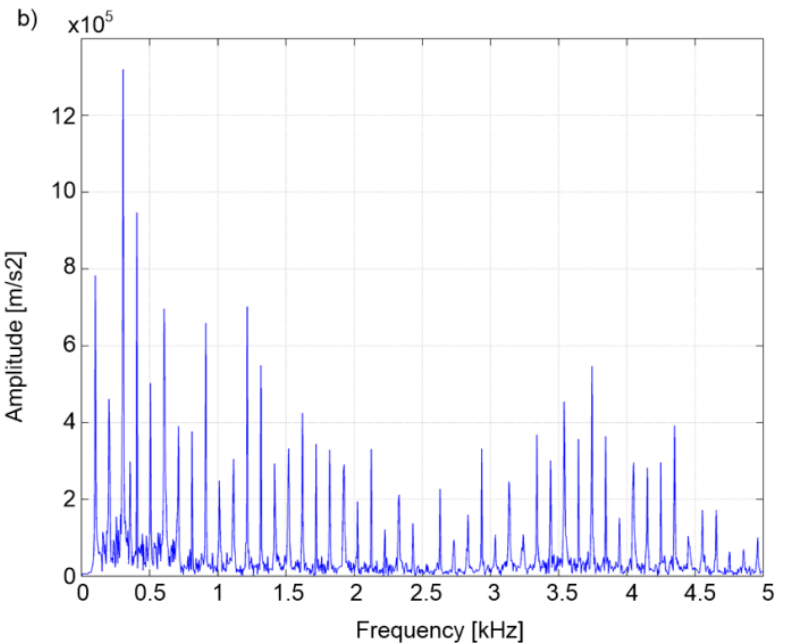

Fig. 2. Spectrum of the vibration signal of the running engine - a) microphone $1, b$ ) accelerometer

Based on the analysis of signals recorded in the last time interval - during the UAV flight, an increase of the disturbance energy for both the microphone and the accelerometer was found. In both cases, the shape of the time waveforms changed. This phenomenon in the case of a microphone is caused by the contribution of additional components of the spectrum of the fluctuation signal of the air streams and gusts of wind.

\section{Conclusions}

The experimental tests carried out allowed to determine the level of vibroacoustic disturbances. The analysis of test results shows that the resultant disturbance signal in the form of noise, complex, multimodal mechanical vibrations of the structure and fluctuation of air streams and gusts of wind cause vibroacoustic disturbances at the level of $140 \mathrm{~dB}$. This means that the acoustic locator mounted on the UAV wing works in conditions of strong interference, the energy of which is concentrated in the band of signals generated by the projectile. The analysis of vibroacoustic disturbances carried out on the basis of registered accelerometer signals indicates that a part of the energy from the band up to $2 \mathrm{kHz}$ is generated by the engine vibration. Research on the mutual correlation of signals from microphones and the accelerometer confirms that the disturbances caused by vibrations of the fuselage, transfer to microphones in the form of vibrations of their membrane.

\section{References}

Panofsky H., Dutton J. (1984). Atmospheric Turbulence: Models and Methods for Engineering Applications. WileyInterscience.

Rodzik D., Grzywiński S., Podciechowski M., Żygadło S. (2014). "The use of LAN-XI measurement modules for recording and acquisition of unmanned aircraft vibroacoustic parameters". Mechanic, No. 7/2014: 637-644.

Grzywiński S., Żygadło S. (2016). "Design of a remote, multi-channel signal recorder". Mechanic, No. 7/2016: 700701.

Grzywiński S., Żygadło S. (2018). "Experimental research of the power supply source parameters for sensors in project management" Proceedings of the 24th International Conference - Engineering Mechanics (EM 2018), ISBN 978-80-214-5497-2, Engineering Mechanics, ACAD SCI Czech Republic 2018 , Pages: 114-117. 